observation revealed congestion of central veins, degeneration of hepatocytes, and reduction of glycogen granules in the DC group. These pathological changes were ameliorated in the flaxseed extract and glibenclamide treated rats.

Conclusion: Flaxseed extract may represent an alternative treatment for the control of diabetes mellitus and its related hepatopathy.

\title{
DISTRIBUTION PATTERN OF HEPATITIS C VIRUS IN EASTERN PENINSULAR MALAYSIA FOR THE PAST DECADE
}

H.M. Hadzri ${ }^{1}$, H.P. Tee ${ }^{2}$ and C.A. Azlida ${ }^{2}$

${ }^{1}$ Department of Medicine, Jalan Hospital Campus, 25150 Kuantan, Pahang. ${ }^{2}$ Gastroenterology Unit, Department of Medicine, Hospital Tengku Ampuan Afzan, Jalan Tanah Putih, 25100 Kuantan, Pahang.

Introduction: Analysis of the Hepatitis C Virus' (HCV) genotypic spread in a particular area has a crucial impact on public health. Genotyping is essential before initiating treatment.

Methodology: This is a hospital-based cohort of 133 chronic hepatitis C patients, collected prospectively among subjects attending Hospital Tengku Ampuan Afzan, Kuantan, within an area of eastern Peninsular Malaysia. We assessed the rate and distribution of HCV genotypes during two consecutive periods, from 2005 to 2006 and from 2013 to 2014, according to age, gender, race and risk factors.

Results: There were more male, Malay ethnic and IDU patients seen in the latter cohort. Genotype 3 was the dominant genotype followed by genotype 1, 4 and 6 . Statistical evaluation of the demographics of HCV patients stratified by genotypes did not reveal any significant difference between the two periods. There was no association between the risk factors analyzed and the acquisition of different HCV genotypes. However, there was significant difference between males and patients receiving treatment in the second cohort $(\mathrm{p}<0.005)$.

Conclusion: The genotype distribution remains similar although there was a trend towards having slightly more genotype 3 in the latter years. The proportions of male patients and patients receiving treatment in the latter period were significantly higher.

\section{DIABETIC RETINOPATHY IN PRIMARY CARE CLINIC USING NON-MYDRIATIC RETINAL CAMERA}

Mohd Aznan Md Aris ${ }^{1}$, Khairidzan Mohd Kamal ${ }^{2}$, Razman Mohd Rus ${ }^{3}$ and Fa'iza Abdullah ${ }^{1}$

${ }^{1}$ Department of Family Medicine, Kulliyyah of Medicine, International Islamic University Malaysia, 25200 Kuantan, Pahang, ${ }^{2}$ Department of Ophthalmology, Kulliyyah of Medicine, International Islamic University Malaysia, 25200 Kuantan, Pahang; ${ }^{3}$ Department of Community Medicine, Kulliyyah of Medicine, International Islamic University Malaysia, 25200 Kuantan, Pahang

Introduction: Diabetic retinopathy is the commonest complication of diabetes mellitus. It is usually detected through funduscopic examination during initial clinical assessment of diabetic patients. For this purpose, the use of retinal cameras in primary care clinics have been introduced for the clinical assessment and diagnosis of diabetic retinopathy.

Objective: To determine the prevalence of diabetic retinopathy through the use of non-mydratic retinal cameras among diabetic patients in government primary care clinics. Its associated risk factors were also evaluated.

Methodology: A cross sectional study was carried out. The subjects were selected through stratified random sampling from among those who attended government primary care clinics in Kuantan, Pahang between May 2010 and April 2011. The subjects were interviewed and assessed clinically using a structured questionnaire. The retinal examination was performed using non-mydratic retinal cameras by trained and accredited staff.

Results: Out of 400 subjects, the majority of them were diagnosed with diabetes mellitus for less than 5 years (58.8\%) and had controlled blood pressure (51.0\%). The prevalence of diabetic retinopathy and maculopathy were $33.5 \%$ and $17.8 \%$ respectively. $22.3 \%$ of scanned retinas had mild non-proliferative diabetic retinopathy. Diabetic retinopathy was independently associated with chronic kidney disease \{OR: 3.46, 95\% CI $(1.76,6.80)\}$ and high HbA1c \{OR: 1.12, 